\title{
GEORREFERENCIAMENTO DE ESPÉCIES ARBÓREAS COMO FERRAMENTA PARA A EDUCAÇÃO AMBIENTAL
}

\author{
Tanny Oliveira Lima Bohner ${ }^{1}$, Cristina Gouvêa Redin ${ }^{2}$, Daniela Thomas da Silva ${ }^{3}$, \\ Cibele Rosa Gracioli ${ }^{4}$, Leopoldo Witeck Neto ${ }^{5}$ \\ ${ }^{1}$ Eng. Florestal, aluna do Curso de especialização em Educação Ambiental pela Universidade Federal de Santa Maria - \\ UFSM. E-mail: tanny.bohner@hotmail.com \\ ${ }^{1}$ Eng. Florestal, Aluna do Programa de pós-graduação em Engenharia Florestal, CCR, Universidade Federal de Santa \\ Maria, RS, Brasil. E-mail: crys luanova@hotmail.com \\ ${ }^{1}$ Eng. Florestal, Aluna do Programa de pós-graduação em Engenharia Florestal, CCR, Universidade Federal de Santa \\ Maria, RS, Brasil. E-mail: com danyzinha th@hotmail.com \\ ${ }^{1}$ Eng. Florestal, Dr. Professora Adjunta da Universidade Federal do Pampa e do Curso de especialização em Educação \\ Ambiental, Universidade Federal de Santa Maria, RS, Brasil. E-mail: cibelegracioli@gmail.com \\ ${ }^{1}$ Eng. florestal, Professor Curso de Paisagismo do Colégio Politécnico, Universidade Federal de Santa Maria, RS, Brasil.
}

\section{RESUMO}

O setor de paisagismo do Colégio Politécnico da Universidade Federal de Santa Maria (UFSM) é um local destinado à coleção de espécies arbóreas com potencial paisagístico e de possível utilização na arborização urbana, jardins e parques. O levantamento, a identificação e o georreferenciamento desses exemplares valorizam e qualificam a paisagem, proporcionando o conhecimento das espécies e sua utilização. Assim, o ambiente torna-se didático e atrativo à comunidade acadêmica e à comunidade em geral. Esse trabalho é de grande importância pela possibilidade de levar as pessoas o conhecimento técnico, promovendo a preservação e conservação ambiental nos espaços verdes públicos e tornando possível a participação individual e coletiva da sociedade na preservação do equilíbrio do meio ambiente. O objetivo desse trabalho foi identificar, rotular e localizar as plantas de pequeno, médio e grande porte, do grupo de árvores (nativas e exóticas), com potencial paisagístico, existentes no setor de paisagismo do Colégio Politécnico da UFSM, em Santa Maria, RS, como uma importante ferramenta de Educação ambiental aos jardins botânicos.

Palavras-chave: identificação, preservação, exemplares arbóreos, paisagismo.

\section{ABSTRACT}

The Polytechnic College landscaping department, of Federal University of Santa Maria (UFSM) is a place reserved to native and exotic tree specimens collection with landscaping potential and 
capable of use in urban landscaping, gardens and parks. The survey, identification and the georeference of these specimens values and qualify the landscape, providing the knowledge of species and its use. Thus, the environment becomes didactic and attractive to the academic community and the general community. This work is very important because of the possibility of providing people the technical knowledge, promoting the preservation and environmental conservation in public green spaces and making it possible for

individual and collective society participation in preserving the environmental balance. The aim of this study was to identify, locate and label the small, medium and large trees (native and exotic), with landscaping potential, at the Polytechnic College landscaping department, in Santa Maria, RS, as an important tool for environmental education at botanical gardens.

Keywords: identification, preservation, tree specimens, landscaping.

\section{INTRODUÇÃO}

Dentre os jardins criados pelo homem, destaca-se o jardim botânico, que possui diversas funções: científica, educacional, social, estética, histórica e ecológica. O jardim botânico apresenta coleções de plantas vivas, ordenadas, documentadas e identificadas, aberto ao público com finalidades de educação, conservação, pesquisa, recreação e prestação de serviços (ROCHA e CAVALHEIRO, 2001).

Esses espaços apresentam coleções de plantas vivas ordenadas, documentadas e identificadas, aberto ao público com finalidades de educação, conservação, pesquisa, recreação e prestação de serviços, exercendo função no desenvolvimento cultural, educacional, científico e econômico (HEYWOOD, 1985; BYE, 1994; ROCHA e CAVALHEIRO, 2001).

O "Jardim" também pode ser visto como um termo universal que adquire conceitos ao longo do tempo e em diferentes culturas como parte do conjunto de espaços livres ou abertos, incluindo tanto os públicos como os privados. E por constituir um termo universal, sua compreensão extrapola territórios na intenção de alcançar sua origem, seu conteúdo filosófico. A essência do jardim parece significar um gesto na paisagem como algo inerente ao convívio do homem com a sociedade em resposta aos seus impulsos. É nessa compreensão que a expressão humana se aproxima mais da arte e da poesia e atinge o conteúdo do paisagismo, em outras palavras, "a arte de fazer jardins" (DOURADO, 1991).

A sociedade, em constante mutação, tem conferido feições diversas às áreas verdes urbanas de uso público ao longo do tempo. Dentre as inúmeras vantagens das áreas verdes, Guzzo (1999) considera três principais: ecológica, estética e social. As contribuições ecológicas ocorrem na medida em que os elementos naturais que compõem esses espaços minimizam tais impactos decorrentes da industrialização. A função estética está pautada, principalmente, no papel de integração entre os espaços construídos e os destinados à circulação. A função social está diretamente relacionada à oferta de espaços para o lazer da população.

De fato, os jardins botânicos são importantes espaços geradores de conhecimento científico, que devem ser socializados para promover reflexões sobre o meio ambiente buscando conscientizar a população sobre a importância da conservação da biodiversidade (CERATI e LAZARINI, 2009).

Segundo Ellison e Ellison (2001), a identificação e rotulagem em muitos jardins botânicos do mundo é deficiente ou inexistente. Por isso, é importante que se faça a devida identificação e 
rotulagem das espécies, principalmente quando elas se encontram em espaços públicos ou em áreas destinadas ao estudo e a pesquisa.

A falta de informações acessíveis à sociedade e a comunidade acadêmica sobre a identificação das espécies vegetais, nas áreas de paisagismo, botânica, dendrologia, silvicultura e conservação da natureza, entre outras, torna a rotulagem necessária como meio de promover o conhecimento e informação nessas áreas. Essa atividade mostra-se eficiente, de baixo custo e altamente acessível, tornando possível a participação individual e coletiva da sociedade na preservação do equilíbrio do meio ambiente como uma importante ferramenta para ligarmos à prática ambiental aos jardins botânicos.

Hoje, um dos principais desafios é interromper o processo de degradação ambiental nas áreas urbanas. Neste sentido, a criação, a recuperação, a qualificação (ou requalificação) dos espaços públicos e de convivência, são fundamentais à sustentabilidade, à valorização da paisagem, à parceria entre instituições públicas e privadas, assim como à melhoria das condições de conforto ambiental. A presença de áreas verdes, urbanas ou não, tem sido objeto de estudos por diferentes grupos e instituições, desde o simples levantamento e mapeamento de espécies (RODRIGUES e GANDOLFI, 1996; GONÇALVES et al., 1998; CARDOSO-LEITE et al,. 1999; MEDEIROS et al.,1999), ou a identificação das espécies com enfoque na educação ambiental, ou trilha ecológica (DIAS et al., 1999), até o resgate do papel social da vegetação para o bem-estar do homem e a melhoria da qualidade de vida (ROCHA e AGRA, 1998; MARENZI, 1999).

Dados de sensoriamento remoto têm sido amplamente empregados na aquisição de informações em aplicações que envolvem grandes extensões territoriais, a exemplo do monitoramento do desflorestamento da Amazônia (INPE, 2007) e dos inventários florestais nacionais (HYYPPA et al., 2000). A capacidade de fornecimento de informações quantitativas precisas, em alta resolução espacial e temporal, torna esse ferramental importante no estudo e gerenciamento da paisagem florestal (FOODY et al., 2003).

O setor de paisagismo do Colégio Politécnico da UFSM é um local destinado a coleção de diversos exemplares arbóreos nativos e exóticos com potencial paisagístico e passíveis de utilização na arborização urbana, jardins e parques. A identificação, a rotulagem e o georefenciamento desses exemplares são importantes para que se conheçam as espécies e seus usos tornando o ambiente didático e atrativo para a comunidade acadêmica e comunidade em geral, promovendo a preservação e conservação ambiental nos espaços verdes públicos.

O objetivo desse trabalho foi identificar rotular e localizar as plantas de pequeno, médio e grande porte, do grupo das árvores (nativas e exóticas) de folhagem perene, com potencial paisagístico, medicinal e ecológico, e ainda alertar sobre potencial tóxico de algumas espécies, existentes no setor de paisagismo do Colégio Politécnico da UFSM, em Santa Maria, RS.

\section{DESENVOLVIMENTO}

O desenvolvimento do trabalho foi feito inicialmente com o levantamento das espécies arbóreas presentes na área de 1 ha. Nesse levantamento foram considerados os caracteres morfológicos tais como: porte da planta, beleza do tronco, aspecto das folhas, presença de frutos e/ou flores, e, grupo paisagístico; seguido de coleta de material botânico, para incorporação ao Herbário do Departamento de Ciências Florestais (HDCFL) - UFSM, bem como consulta a bibliografia especializada e/ou profissionais da área para posterior identificação. 
A segunda etapa constou da identificação ou rotulagem dos indivíduos utilizando-se etiquetas de plástico (Figura 1), na cor branca, e com o auxílio de um lápis tipo "Dermatograph" foram registradas a espécie e a família das plantas pertencentes ao acervo. Estas etiquetas foram presas ao caule dos exemplares com fio de algodão, em uma altura média de 0,50 $\mathrm{m}$ a 1 $\mathrm{m}$ (nas plantas com mais de $1 \mathrm{~m}$ ), e, rente ao solo nas plantas com menos de $1 \mathrm{~m}$, no sentido sul de orientação. No momento da identificação, foi feito o registro fotográfico de cada espécie, para incorporação do mesmo na ficha do herbário. As plantas foram identificadas, rotuladas e fotografadas, sendo apresentadas com suas características botânicas, segundo Longhi (1995), Backes e Irgang (2002) e Carvalho (2003).

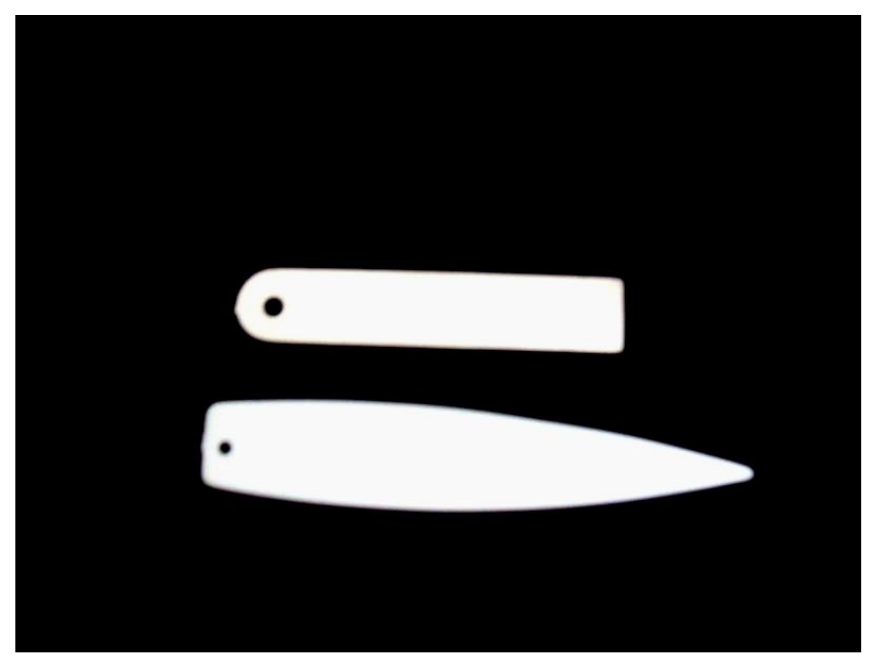

Figura 1 - Aspecto das placas de identificação utilizadas na catalogação das espécies vegetais. Fonte: (Bohner, 2010)

Após a identificação com as etiquetas, de forma bastante visível, procedeu-se o georeferenciamento de cada exemplar arbóreo com auxílio de GPS do tipo GARMIN, que posteriormente teve suas informações, ou seja, as coordenadas de cada ponto capturado, descarregadas no software Spring 5.1. Para tal, foram utilizados o sistema de coordenadas geográficas (LAT/LONG) e o Datum WGS 1984. A finalidade dessa atividade foi a confecção de croqui explicativo de situação, da área do jardim, que poderá ser visualizada na entrada do mesmo com a colocação de uma placa explicativa com os principais pontos e espécies mais importantes do acervo, para que a população tenha a oportunidade de fazer uma visita de forma guiada, reconhecendo as espécies nativas e exóticas comumente utilizadas nas áreas verdes.

Todos os materiais botânicos coletados foram arquivados no acervo do Herbário Florestal UFSM, com a finalidade de comprovar a identidade do material e servir para consultas futuras sobre dúvidas na nomenclatura das espécies. Uma vez que as pessoas visitem o jardim, terão também a possibilidade de visitar o herbário onde essas espécies estarão herborizadas e com maior detalhamento botânico. Existe ainda a possibilidade futura da confecção de folders explicativos com o histórico da área do jardim (setor de paisagismo do Colégio Politécnico da UFSM), bem como a descrição ilustrada das principais espécies nativas e exóticas para o conhecimento da comunidade. O período de desenvolvimento do trabalho foi de fevereiro a agosto de 2010. 


\section{RESULTADOS}

As espécies foram identificadas, rotuladas, fotografadas e georeferenciadas para confecção de croqui (Figura 2).

As famílias botânicas das espécies arbóreas nativas da categoria das folhosas tropicais e temperadas que foram identificadas e catalogadas na área foram: Anacardiaceae, Anonaceae, Apocinaceae, Aquifoliaceae, Araliaceae, Bignoniaceae, Boraginaceae, Euphorbiaceae, Fabaceae, Lamiaceae, Lauraceae, Lythraceae, Malvaceae, Melastomataceae, Meliaceae, Myrtaceae, Moraceae, Myrsinaceae, Proteaceae, Rosaceae, Rutaceae, Salicaceae, Santalaceae, Sapindaceae, Sapotaceae. As famílias de espécies folhosas exóticas foram: Altingiaceae, Apocynaceae, Anacardiaceae, Araliaceae, Adoxaceae, Casuarinaceae, Euphorbiaceae, Fabaceae, Fagaceae, Ginkgoaceae, Lauraceae, Lythraceae, Magnoliaceae, Malvaceae, Meliaceae, Myrtaceae, Moraceae, Oleaceae, Platanaceae, Rosaceae, Rutaceae, Salicaceae, Sapindaceae, Theaceae.

Quanto ao potencial paisagístico pode-se dizer que a maioria das espécies pertencentes a essas famílias e categorias, apresentaram-se apropriadas para arborização urbana e/ou composições paisagísticas em pequenos, médios e grandes espaços (considerando critérios adequados ao espaçamento), proporcionando sombra, beleza da folhagem, beleza das flores (atrativos a insetos). Algumas espécies nativas que podem ser citadas são: Handroanthus heptaphyllus (Ipê-roxo) Handroanthus chrysotrichus (Ipê-amarelo), Jacaranda micrantha (Jacarandá-mimoso), Senna multijuga (Chuva-de-ouro), Tibouchina mutabilis (Quaresmeira), Casearia sylvestris (Chá-de-bugre) e Allophylus edulis (Chal-chal); e as espécies exóticas Delonix regia (Flamboyant), Liriodendron tulipifera (Tulipeira), Hibiscus mutabilis (Malva-rosa) e Prunus serrulata (Cerejeira-japonesa).

Quanto ao aspecto ecológico à presença de frutos mostra-se importante principalmente quanto a atração da avifauna, e nesse caso as arbóreas nativas são as mais importantes, são elas: Annona sylvatica (Araticum), Acca sellowiana (Goiaba-da-serra), Campomanesia Xanthocarpa (Guabiroba), Eugenia involucrata (Cerejeira), Eugenia uniflora (Pitangueira) e Psidium cattleianum (Araçá).

Em relação ao potencial medicinal podem-se citar as nativas: Ilex paraguaryensis (Ervamate), Bauhinia forficata (Pata-de-vaca), Campomanesia guazumifolia (Sete-capotes) Ocotea puberula (Canela-guaicá), Casearia sylvestris (Chá-de-bugre) e Jodina rhombifolia (Cancorosa). E as exóticas: Ginkgoaceae (Ginkgo biloba - Ginkgo) e Lauraceae (Cinnamomum zeylanicum - Canela).

As espécies nativas que apresentam potencial tóxico ou alérgico são principalmente algumas das famílias Anacardiaceae (Lithraea brasiliensis - Aroeira-brava), Apocynaceae (Aspidorsperma parvifolium - Peroba-vermelha) e Euphorbiaceae (Sebastiana commersoniana Branquilho). As famílias e espécies das exóticas com potencial tóxico são: Apocynaceae (Nerium oleander - Espirradeira; Plumeria rubra - Jasmim-manga), Euphorbiaceae (Aleurites fordii Tungue), Oleaceae (Ligustrum Lucidum - Ligustro), Rhamnaceae (Hovenia dulcis - Uva-do-japão). 


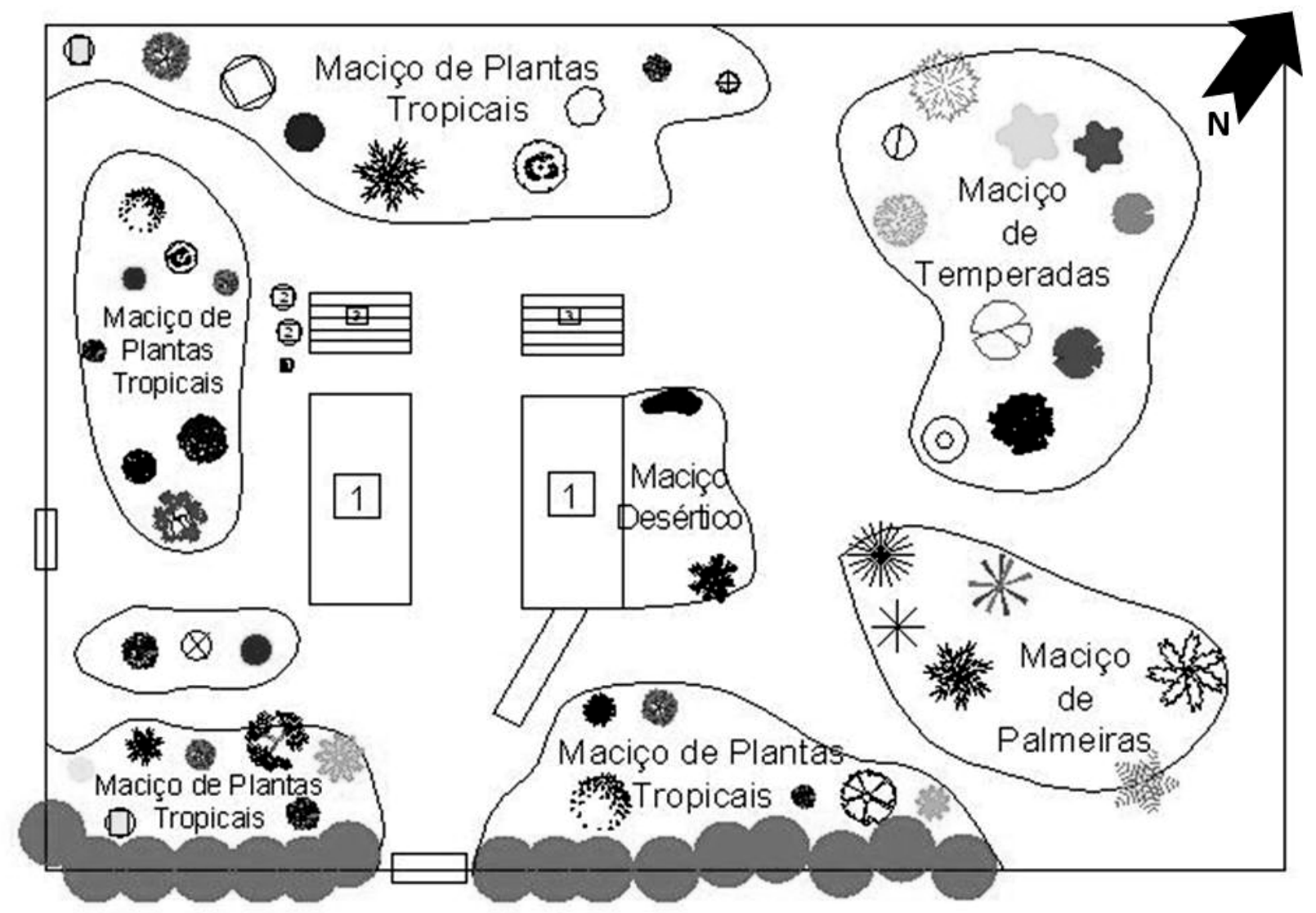

Figura 2 - Croqui esquemático da área de estudo, setor de paisagismo, Colégio Politécnico, UFSM, Santa Maria, 2010. 

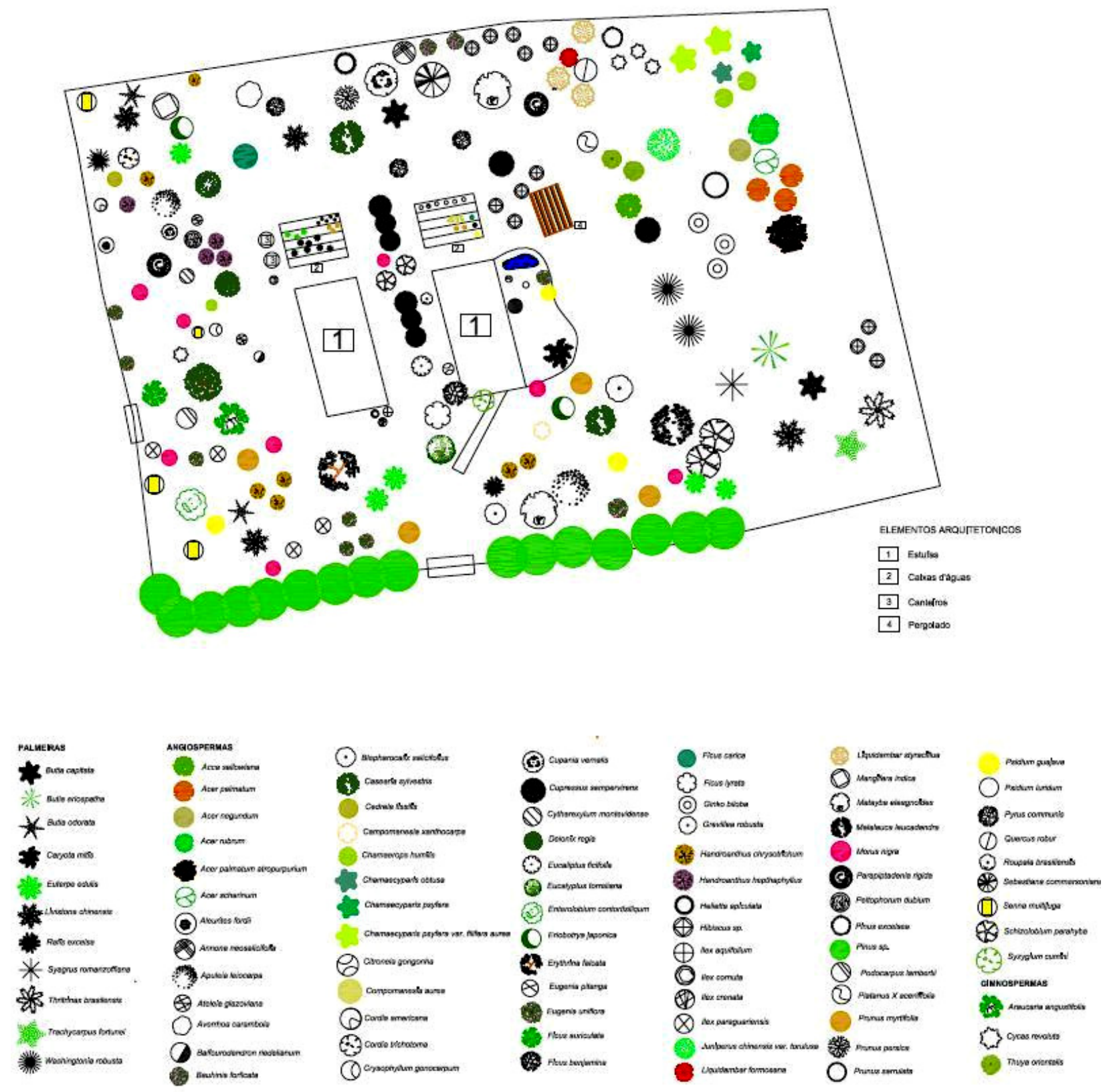

Figura 2 - Espécies arbóreas georreferenciadas na área de estudo, setor de paisagismo, Colégio Politécnico, UFSM, Santa Maria, 2010.

\section{CONCLUSÕES E RECOMENDAÇÕES}

Conclui-se que esse trabalho é de grande importância pela possibilidade de levar as pessoas o conhecimento técnico, de fundamental importância ao ambiente, uma vez que o Setor de Paisagismo do Colégio Politécnico abriga uma extensa coleção de espécies arbóreas nativas e exóticas, com potencial paisagístico, importância ecológica e medicinal e que na maioria das vezes 
não é apresentado de forma acessível à comunidade acadêmica e comunidade em geral. Recomenda-se que o trabalho de conscientização tenha uma continuidade, instruindo sobre a importância do mesmo e alertando a população sobre riscos eminentes de toxidez ou alergia de determinadas espécies, bem como os benefícios da maioria das árvores. Somente com o conhecimento desses elementos será possível a efetiva preservação e conservação da vegetação nas áreas públicas e coletivas aumentando a consciência ambiental das pessoas.

\section{REFERÊNCIAS BIBLIOGRÁFICAS}

BACKES, P; IRGANG, B. Árvores cultivadas no Sul do Brasil: Guia de identificação e interesse paisagístico das principais espécies exóticas. Ed. Paisagem do Sul, 2004; 204 p.

BYE, R. 1994. Historia de los jardines botanicos: evolucion de estilos, ideas y funciones. Chapingo (Serie Horticultura) 2:43-53.

CARDOSO-LEITE, Eliana et al. Mapeamento da Vegetação de uma Reserva Biológica no Sudeste do Brasil, Através de Fotointerpretação e Levantamento de Campo, Como Subsídio ao seu Zoneamento e Conservação. In: CONGRESSO NACIONAL DE BOTÂNICA, 50, 1999, Blumenau/SC. Programa e resumos... Blumenau: Soc. Bot. Br., 1999. P. 149.

Carvalho, P. E. R.; Espécies arbóreas brasileiras. Ed. Embrapa Informação Tecnológica, 2003, 1035 p.

CERATI, T. M.; LAZARINI, R. A. M. A pesquisa-ação em Educação Ambiental: Uma experiência no entorno de uma unidade de Conservação Urbana. Revista Ciência e Educação. V. 15, n. 2, p. 383-92, 2009.

DIAS, A. et al. Programa piloto de educação ambiental na trilha do Jacatirão, Ilha de Santa Catarina, Florianópilos. In: CONGRESSO NACIONAL DE BOTÂNICA, 50, 1999, Blumenau/SC. Programas e recursos... Blumenau: Soc. Bot. Br., 1999, p. 51

DOURADO, G. M. Burle Marx: O prazer de viver e trabalhar com a natureza. (Entrevista). Revista Projeto. n.146, p.58-77. São Paulo, 1991.

ELLISON D.; ELLISON A."Cultivated Palms of the World".Ed. UNSW2001;pgs. 257.

FOODY, G.M.; Boyd, D.S.; Cutler, M.E.J. 2003. Predictive relations of tropical forest biomass from Landsat TM data and their transferability between regions. Remote Sensing of Environment, 85(4): 463-474.

GONÇALVEZ, F. C. C. ET. AL. Análise Pós-ocupação de uma praça de Burle Marx em Recife. In: CONGRESSO NACIONAL DE BOTÂNICA, 49, 1998, Salvador/BA. Resumos... Salvador: Soc. Bot. Br., 1908. P. 265.

GUZZO, P. Estudos dos espaços livres de uso público e da cobertura vegetal em área urbana da cidade de Ribeirão Preto-SP. 1999. 106f. Dissertação (Mestrado em

Geociências) . Instituto de Geociências e Ciências Exatas, Universidade Estadual Paulista, Rio Claro. 1999.

HEYWOOD, V.H. 1985. Botanic gardens conservation strategy. World Wide Fund for Nature, Gland. 
Monografias Ambientais (e-ISSN: 2236-1308)

HYYPPA, J.; HYYPPA, H.; INKINEN, M.; ENGDAHL, M.; LINKO, S.; ZHU, Y. 2000.

Accuracy comparison of various remote sensing data sources in the retrieval of

forest stand attributes. Forest Ecology and Management, 128(1-2): 109-120.

INPE (Instituto Nacional de Pesquisas Espaciais), 2007. Projeto PRODES:

monitoramento da floresta amazônica brasileira por satélite. Disponível

em: http://www.obt.inpe.br/prodes/. Acesso em: 18 abr. 2010.

LONGHI, R. A. Livro das árvores e arvoretas do Sul. Ed. L \& PM, 1995; 167 p.

MARENZI, R. C. A Influência da Vegetação nas Preferências Paisagísticas no Municípioda Penha. In: CONGRESSO NACIONAL DE BOTÂNICA, 50, 1999, p. 150.

MEDEIROS, J.de D. et. al. A vegetação o Parque Florestal do Rio Vermelho. In: CONGRESSO NACIONAL DE BOTÂNICA, 50, 1999, Blumenau/SC. Programa e resumos... Blumenau: Soc. Bot. Br., 1999. P. 150

ROCHA, E. A. e AGRA, M. e F. Cactaceae Medicinais a Paraíba, Brasil. In: CONGRESSO NACIONAL DE BOTÂNICA, 49, 1998, Salvador/BA. Resumos... Salvador: Soc. Brot. Br., 1998, p. 285.

ROCHA, Y. T. e CAVALHEIRO, F. Aspectos históricos do Jardim Botânico. Revista Brasileira de Botânica.

RORIGUES, R. R.; GANDOLFI, S. Recomposição de florestas nativas: princípios gerais e subsídios para uma definição metodológica. Revista brasileira de Horticultura Ornamental, Campinas, c. 2, n. 1, p. 4-15, 1996. 